\section{Prostate-bound nanoparticles}

\section{By Kai-Jye Lou, Staff Writer}

Platinum drugs such as cisplatin, which have been successfully used for the treatment of different types of cancer, are notoriously inefficient with prostate cancer, due mostly to the particular physiology of the prostate and the chemistry of the drugs themselves. Now, researchers at the Massachusetts Institute of Technology and Harvard Medical School have engineered a low-toxicity, prostate-specific nanoparticle containing a cisplatin prodrug that is efficacious in resistant prostate cancer cell lines. ${ }^{1}$

When delivered systemically, drug toxicities can limit the amount of cisplatin that is given, and such dosing is often not sufficient for effective treatment. Sub-therapeutic doses also can lead to resistance. In addition, cisplatin is hydrophilic, which makes it difficult for use with most controlled-release hydrophobic drug delivery vehicles.

In a paper published in the Proceedings of the National Academy of Sciences, the research team describes a cisplatin prodrug that is hydrophobic and thus more amenable to use with controlledrelease PEGylated nanoparticles with hydrophobic polymer poly(D,L-lactic-co-glycolic acid) (PLGA) cores. ${ }^{1}$

The prodrug is loaded into the hydrophobic core of the nanoparticles. The hydrophilic polyethylene glycol (PEG) component surrounds the hydrophobic core, creating a water shield that prevents the host immune system from detecting and destroying the nanoparticles. Finally, the whole complex is coated with an aptamer that targets cells expressing prostate-specific membrane antigen (PSMA).

Omid Farokhzad, a coauthor on the PNAS paper and an assistant professor of anesthesiology at Harvard Medical School, told SciBX, "The chemists in the team developed a hydrophobic cisplatin prodrug that is reduced in an intracellular environment to become cisplatin. The aptamer-coated nanoparticle carries the prodrug to cells expressing PSMA and gets internalized via receptor-mediated endocytosis."

In his earlier work, Farokhzad had shown that varying the ratios between PLGA, PEG and aptamer can modulate nanoparticle physicochemical properties such as size and the release profile of the drug payload. ${ }^{2} \mathrm{He}$ had also shown that the aptamer-based, PSMA-targeting nanoparticles can be loaded with unmodified docetaxel and used to treat prostate cancer in mice. ${ }^{3}$

In the current paper, experiments with two human prostate adenocar- cinoma cell lines showed that resistant $\mathrm{PSMA}^{+}$cells efficiently incorporate the nanoparticles, whereas PSMA ${ }^{-}$cells do not.

In the resistant $\mathrm{PSMA}^{+}$adenocarcinoma cells, the targeted nanoparticles had an $\mathrm{IC}_{50}$ value of $0.03 \mu \mathrm{M}$ compared with $0.13 \mu \mathrm{M}$ for nontargeting nanoparticles and $2.4 \mu \mathrm{M}$ for free cisplatin.

According to Stephen Lippard, a coauthor on the PNAS paper and professor of chemistry at MIT, the aptamer-targeted nanoparticle drug delivery system prolongs the half-life of cisplatin and the high tissue specificity increases the chance of delivering a large dose of the drug to the tumor.

"Conceptually, that's what you want to do," Lawrence Tamarkin, president, $\mathrm{CEO}$ and cofounder of CytImmune Sciences Inc., told SciBX. "You want to have a water shield to protect the molecule and a molecule to target the tumor."

CytImmune is developing a Aurimune, a tumor necrosis factor-bearing PEGylated colloidal gold nanoparticle that has completed Phase I testing in solid tumors. The company plans to start a Phase II trial in 1Q09.

\section{Made for one another}

Farokhzad told SciBX that "past work in this area of targeted drug delivery has focused on one of two strategies: you could engineer a vehicle to accommodate the drug or you could engineer a drug to be suitable for the vehicle. Our current work does both. It's basically a marriage of the two approaches."

The result, Farokhzad said, is "tunable and controlled release of most therapeutics, which would be hard to do with a liposome-based delivery system." He said the flexibility of the nanoparticle approach makes it a good drug delivery platform that can incorporate a variety of drug types.

"A key theoretical advantage for aptamers in this context is their chemical nature and the ability to use diverse chemical conjugation strategies and conditions to build a designed entity," said Page Bouchard, SVP of discovery and preclinical development at Archemix Corp. "This is a distinct advantage over biological-based targeting entities, which have far more restrictive chemical properties and design options."

Archemix's lead compound is ARC1779, an antiplatelet aptamer in Phase II testing to treat thrombotic thrombocytopenic purpura (TTP).

"This principle of PSMA aptamer-mediated targeted delivery to prostate cancer cells has now been demonstrated for cisplatin- and docetaxelcontaining nanoparticles," Bouchard told SciBX. "We therefore believe that PSMA-binding aptamers as a prostate cancer-targeted delivery strategy holds great potential."

\section{Optimizing delivery}

Farokhzad noted that it still would be necessary to optimize individual parameters of the system. "For example," he said, "if we find that the 
aptamer triggers an immune response, we will have to switch it out for another one."

CytImmune's Tamarkin said the technology published in the PNAS article is "clever by design, but they need to make sure the particle is invisible to the immune system." He said nanoparticle-based delivery systems need to have the correct ratio of PEG to create a water shield that can evade the immune system.

Another consideration is nanoparticle size. The PNAS paper notes that particles larger than $200 \mathrm{~nm}$ are generally ineffective in vivo. The nanoparticles used by Lippard's group were about $140 \mathrm{~nm}$.

Tamarkin wanted to know whether these nanoparticles will remain small enough in the bloodstream, where apparent size can increase significantly because of the nanoparticle's physical and chemical properties such as shape and degree of hydration.

Regardless of what happens in the blood, Farokhzad said aptamers have a size advantage over biologics such as antibodies.

"Size matters-antibodies are large molecules around $15 \mathrm{~nm}$ or so, and if you start to decorate the surface of the nanoparticle with antibodies, you quickly increase its size," he said. "Aptamers on the other hand, are only 2-3 nm. You want to keep the particles as small as possible."

Tamarkin thinks manufacturing will be one of the main challenges. He noted that issues with product consistency can arise when the manufacturing process is complex. "In Dr. Lippard's paper, the nanoparticle manufacturing process requires many organic synthesis steps. We need to know if this is a scalable process," he said.

Bouchard thinks the steps needed to get the nanoparticle prodrug technology into the clinic include "validation of these pharmacology studies, development of a viable manufacturing method for the combined entity and the conduct of standard preclinical regulatory development studies."
Lippard said a major concern is whether or not the drug payload will be properly delivered in an in vivo setting. "The one thing we want to avoid is the premature release of platinum in blood, before the nanoparticle enters the cancer cells," he said.

Lippard said the next steps will be moving the nanoparticle candidate into animal studies.

Farokhzad added that it will be important to compare the efficacy and tolerability of escalating doses of the prodrug-containing nanoparticle to free cisplatin.

Finally, he said pharmacokinetics and biodistribution studies will be required. "Clearly, the nanoparticles are taken up by the cells," he noted, "but are there any nonspecific cell effects or uptake by other cell types?"

Lippard said a provisional patent has been filed covering the targeted platinum drug delivery system. Licensing details are available from the MIT Technology Licensing Office.

Lou, K.-J. SciBX 1(41); doi:10.1038/scibx.2008.988

Published online Nov. 13, 2008

\section{REFERENCES}

1. Dhar, S. et al. Proc. Natl. Acad. Sci. USA; published online Oct. 20, 2008; doi:10.1073/pnas.0809154105

Contact: Stephen J. Lippard, Massachusetts Institute of Technology, Cambridge, Mass.

e-mail: lippard@mit.edu

Contact: Omid C. Farokhzad, Harvard Medical School, Boston, Mass. e-mail: ofarokhzad@zeus.bwh.harvard.edu

2. Gu, F. et. al. Proc. Natl. Acad. Sci. USA 105, 2586-2591 (2008)

3. Farokhzad, O.C. et. al. Proc. Natl. Acad. Sci. USA 103, 6315-6320 (2006)

\section{COMPANIES AND INSTITUTIONS MENTIONED}

Archemix Corp., Cambridge, Mass.

CytImmune Sciences Inc., Rockville, Md.

Harvard Medical School, Boston, Mass.

Massachusetts Institute of Technology, Boston, Mass. 